\title{
Practicum y Trabajo de Fin de Master como ejes profesionalizadores: \\ Análisis de un Master Erasmus Mundus
}

UT. Revista de Ciències de l'Educació

Desembre 2014. Pag. 49-73

ISSN 1135-1438. EISSN 2385-4731

http://pedagogia.fcep.urv.cat/revistaut

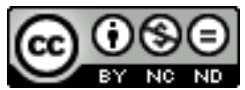

\section{Charo Barrios Arós a , Pilar Iranzo Garcíab i Juana Maria Tierno García ${ }^{\text {c }}$ \\ Rebut: 28/10/2014 Acceptat: 21/11/2014}

\section{Resumen}

El objetivo de este artículo es analizar cómo el diseño del Practicum y del Trabajo de Fin de Master (TFM) pueden favorecer procesos de profesionalización e internacionalización, en un master Erasmus Mundus.

Se presentan diferentes concepciones asociadas tradicionalmente a la formación práctica (Practicum) a las que se suman visiones actuales sobre colaboración interinstitucional entre universidades y centros formadores, participación de diferentes países (internacionalización) y, con ello, promoción de procesos de interculturalidad, así como vinculación con dinámicas de investigación sobre la propia práctica profesional. El TFM juega un papel central en estas aportaciones.

Se utiliza una metodología descriptiva y de estudio de caso para analizar desde estos parámetros el Master Erasmus Mundus de "Formación de profesionales de la formación".

Los resultados del análisis ponen de manifiesto la importancia de un diseño holístico que favorezca la profesionalización como implicación en la resolución de problemáticas reales de carácter internacional, y considere la evaluación y la metaevaluación de la globalidad del proceso como una parte intrínseca de esa misma profesionalización.

Palabras clave: Practicum, Trabajo Fin de Master, competencias, profesionalización, internacionalización, interculturalidad, investigación sobre la propia práctica, master Erasmus Mundus.

\footnotetext{
${ }^{a}$ Departament de Pedagogía. Universitat Rovira i Virgili - Tarragona

${ }^{\mathrm{b}}$ Departament de Pedagogía. Universitat Rovira i Virgili - Tarragona

${ }^{c}$ Departament de Pedagogía. Universitat Rovira i Virgili - Tarragona
} 


\section{Abstract}

The aim of this paper is to analyze how design of Practicum and Final Project Master (FPM) can promote professionalization and internationalization processes in an Erasmus Mundus master.

We present different concepts traditionally associated to practical training (Practicum) but we add currents views on interagency collaboration between universities and training centers, involving different countries (internationalization) and promoting intercultural processes, as well as linking with research dynamics on own professional practice. The TFM plays a central role in these contributions.

We used a descriptive methodology and the case study to analyze these parameters from the Erasmus Mundus Master "Training of training professionals".

The analysis results highlight the importance of a holistic design to promote professionalization, as involvement in solving real problems of an international character, and that considers the evaluation and meta-evaluation of the all process as an intrinsic part of the same professionalization.

Keywords: Practicum, Final Project Master, competences, professionalization, internationalization, multiculturalism, research on own practice, Erasmus Mundus master.

\section{Introducción ${ }^{1}$}

Una de las grandes finalidades de las instituciones que se ocupan de la formación de futuros profesionales es que éstos aprendan a realizar las funciones propias de la profesión. En el logro de este objetivo, el Practicum ha desempeñado un papel fundamental, permitiendo al estudiante ponerse en contacto directo con la realidad laboral y con los quehaceres del ámbito profesional para el que se está formando. Entre otros, se han destacado como aportes profesionalizadores, el conocimiento situado, la resolución de problemas profesionales reales y la colaboración con comunidades de aprendizaje (Méndez, 2012), así como los procesos de problematización y de transacción implicados en la profesionalización (Jorro, 2007).

En la actualidad, teniendo presente el movimiento de transformación generado con la Declaración de Bolonia y el marco del EEES, las universidades europeas reflexionan sobre los modelos formativos basados en las competencias profesionales (en España, destacan las sucesivas ediciones del Symposium Internacional sobre Practicum que se celebran en Poio - Pontevedra) y sobre cómo acomodar el Practicum a los parámetros organizativos y docentes de la convergencia (Tejada, 2005; Zabalza, 2007, 2011). Éste se erige como lugar de intersección o conexión entre el ámbito laboral y el ámbito formativo, un espacio privilegiado de integración y encuentro entre la teoría y la práctica desde el enfoque de las competencias. 
Por otra parte, el EEES articula los niveles de grado y postgrado y caracteriza este último como profesionalizador, incorporando, también y complementariamente, competencias de reflexión sobre la práctica e investigadoras.

En directa correlación con los estudios de grado, los de master también incluyen en su planificación curricular la elaboración y defensa pública del Trabajo de Fin de Master, de entre 6 y 30 créditos (MEC, 2007). Este trabajo, compendio de los aprendizajes durante todo el master, ha de consistir en un "conjunto de explicaciones, teorías, ideas, razonamientos y apreciaciones sobre una temática concreta escogida entre tutor/a y estudiante" (AQU, 2013). El tema de estudio generalmente entronca con las líneas de investigación de los departamentos o de las facultades donde se realiza $y$, siguiendo las recomendaciones europeas, debe poder transferirse a las empresas, a la sociedad o a otras investigaciones.

En el ámbito europeo, uno de los programas académicos más relevantes es el Master Erasmus Mundus (http://eacea.ec.europa.eu/erasmus_mundus), pensado, entre otras razones, para fortalecer la cooperación entre universidades europeas (a través de consorcios) y la formación de estudiantes europeos y no europeos en ámbitos de interés internacional.

En este trabajo pretendemos analizar cómo el diseño del Practicum y el Trabajo de Fin de Master (TFM) pueden favorecer procesos de profesionalización e internacionalización en un master Erasmus Mundus. Nuestro marco conceptual atiende a las características propias del diseño, desarrollo y evaluación de la formación práctica como base de procesos formativos profesionalizadores, a partir de la investigación de la práctica profesional en contextos internacionales e interculturales. Los referentes más cercanos se hallan en la literatura que, desde los años 80 , analiza la formación práctica para la enseñanza. Nos basamos en ellos para realizar un ejercicio de transferencia entre esas bases y el reto actual de articular Practicum y TFM altamente profesionalizadores.

El diseño del mismo apunta a un modelo de organización que puede orientar futuros diseños de prácticas y trabajo final de estudios, sean de grado o de master ya que, como se verá, el énfasis se pone en la estrecha conexión entre ellos y en cómo éstos influyen en la fase de profesionalización que cabalga entre la formación inicial y el ejercicio profesional.

\section{El Practicum y el Trabajo de Fin de Master como ejes profesionalizadores}

Con anterioridad a la incorporación de los Trabajos Fin de Grado (TFG) y Trabajos Fin de Master (TFM) como elementos centrales en los planes de estudios universitarios, Zabalza (2004) señaló cuatro tipos de condiciones para el desarrollo óptimo de programas de Practicum. Estas condiciones siguen siendo imprescindibles en el contexto actual, en el que la formación de master acredita la adquisición por parte 
del estudiante de aquellas competencias profesionalizadoras 'especializadas', incluyendo las relacionadas con la investigación.

\section{Condiciones que determinan el sentido formativo}

Para que el Practicum adquiera pleno sentido formativo ha de planificarse teniendo en cuenta que es un periodo de aprendizaje y, como tal, debe aportar nuevos inputs y oportunidades de formación a los estudiantes. Schön (1998) sitúa al Practicum en una posición intermedia entre la formación teórica que se imparte en las aulas y el mundo de la práctica real.

En el marco del EEES, se ha debatido entre la conveniencia de una formación más centrada en las competencias propias de la profesión o la de una formación general y de base. Si se opta por modelos formativos centrados en competencias específicas, el Practicum será concebido como un componente más del periodo de formación al que se encomiendan aprendizajes concretos. Los modelos centrados en el desarrollo general y polivalente ofrecerán modalidades de prácticas en las que cada estudiante se centre en su propio itinerario formativo.

En cualquier caso, la mayoría de las universidades han integrado el TFM en la formación práctica de master, concebido como un trabajo de investigación. Se hace énfasis en una competencia del futuro profesional, la reflexivo-investigadora, a la que hasta hace relativamente poco se concedía escasa importancia para el ejercicio de la profesión, por lo que se abordaba someramente. De ahí que entendamos el Practicum y el TFM como las dos caras de un mismo proceso formativo.

\section{Condiciones curriculares}

Están referidas a la forma en que debe integrarse en el conjunto del Plan de estudios. Según Zabalza (2004), la dimensión curricular del Practicum podría articularse en torno a: La formalización del programa de prácticas, la integración del Practicum en el proceso global de la carrera, las fases en las que está establecido, las estrategias de supervisión adoptadas durante el Practicum y los dispositivos de evaluación arbitrados.

\section{Condiciones organizativas}

Son las relativas a la forma en que debe ser planteada la relación entre las instituciones y el desarrollo del proceso.

Las características del escenario de prácticas. Para la selección de los centros de prácticas, es necesario considerar que los efectos formativos de éstas, varían en función de las características materiales y técnicas (tamaño, ubicación, tipo de actividad desarrollada, recursos, etc.) y del estilo de funcionamiento y cultura 
'institucional' (clima de trabajo, hábitos de cooperación, etc.) de los escenarios donde se realizan.

El estilo de colaboración interinstitucional. Para que Practicum y TFM se desarrollen con éxito, la relación universidad-centro de prácticas ha de estar encaminada al fin común que es la formación de profesionales y basarse en una actitud de apertura hacia las aportaciones fundamentadas que realicen los estudiantes para la mejora.

\section{Condiciones personales}

Para ejercer la tutoría y ayudar al futuro formador en su desarrollo, es preciso que el tutor amplíe y mejore sus propias competencias personales y participativas, ejercite su inteligencia interpersonal e intrapersonal (Gardner, 1998), así como la emocional (Goleman 1996, 2005) y lo que Denzin (2007) denomina 'comprensión emocional'. Es decir, que aprenda a crear confianza de una manera que 'toque' el núcleo emocional y la identidad de los formadores en un contexto de responsabilidad (Orland-Barak, 2006).

\section{Modelos de prácticas}

Es lógico pensar que cada institución tiene su modelo de prácticas, por lo que se han hecho diversos intentos de clasificación de los mismos priorizando unas características sobre otras. En nuestro contexto es muy conocida y utilizada la de Zabalza y Marcelo (1993) que recoge cuatro modelos básicos para la formación de docentes, trasladables a otros ámbitos de la formación. En este momento, sólo los mencionamos: modelo tradicional o de oficio, modelo de laboratorio o simulación, modelo reflexivo y modelo de prácticas basado en la investigación.

Aunque tradicionalmente se viene aludiendo a esos modelos, aparece con fuerza la idea de un modelo holístico (Domingo y Fuertes, 2007) que plantea el Practicum como eje vertebrador de las materias y en el que confluyen la mayoría de las asignaturas de la titulación con el fin de interrelacionar la teoría con la práctica educativa. Crean para tal fin una estructura compleja que proporciona una visión global, unitaria e integradora de los distintos elementos de la realidad estudiada, nacida del esfuerzo y de la colaboración de docentes y tutores. Global, porque el futuro profesional de la formación ha de intervenir en un escenario natural que no admite la fragmentación del conocimiento. Unitaria e integradora, porque su intervención es una acción concreta que sintetiza la diversidad de conocimientos teóricos y experienciales y sus interacciones.

Esta estructura va creciendo en complejidad paulatinamente y permite al estudiante establecer relaciones cognitivas y experienciales, interdisciplinares e intradisciplinares crecientes, abiertas y creativas, llevándole a construir una síntesis profesionalizadora en su saber, saber hacer y saber estar. 
Estas mismas autoras manifiestan que dicho enfoque curricular conduce a una mayor relación entre el conocimiento teórico y el práctico. Por otro lado, viene a superar el planteamiento tradicional de estricta complementariedad entre teoría y práctica, tan extendido en el ámbito académico universitario.

Cabe resaltar la importancia de la colaboración entre la universidad y los centros de prácticas. Los diversos modelos de colaboración responden a una determinada manera de entender el proceso de formación, es decir de entender cómo se aprende, cómo se enseña y cómo se aprende a actuar profesionalmente. Los modelos que, en el contexto de la formación docente, han sido identificados al analizar las complejas relaciones entre estas instituciones son los siguientes (Cochran-Smith, 1999; Marcelo y Estebaranz, 1998; Vaillant y Marcelo, 2001):

\section{Modelo de Yuxtaposición}

Considera el Practicum como el elemento curricular más importante en la formación del profesional, pues es donde se demuestra su competencia. Su filosofía se resume en: "si quieres llegar a ser buen profesional, haz lo que los buenos profesionales hacen". El estudiante en formación aprende a actuar observando cómo lo hacen los profesionales $y$, posteriormente, imitándolos. El experto profesional enseña al estudiante su propio estilo, es decir, sus destrezas, actitudes y rasgos de personalidad y el estudiante lo asimila a través de la observación, la imitación y la práctica dirigida.

Este modelo es el que se asemeja más al modelo tradicional o de oficio, aunque pone de relieve la necesidad de unos conocimientos teóricos previos que deben ser adquiridos en la universidad (perspectiva académica, conceptual y proposicional). Después se desarrollarán las prácticas en los centros donde el estudiante aplicará los conocimientos ya adquiridos. Corresponde al estudiante establecer las posibles conexiones entre la teoría (curriculum explícito cursado en la universidad) y la práctica (curriculum vivido durante las prácticas). Por lo tanto, el nivel de colaboración entre las dos instituciones puede ser mínimo.

Otras críticas de este modelo son: que favorece un tipo de aprendizaje pasivo por parte de los estudiantes, que ofrece una perspectiva parcial del ejercicio profesional al no permitir ir más allá de aquello que se observa y que delega la formación del estudiante en la 'buena voluntad' del tutor a quien, hasta ahora, no se le ha exigido una formación específica para desempeñar tareas de tutorización.

\section{Modelo de Consonancia}

Cochran-Smith (1999) tomó el término de 'consonancia' empleado en el campo de la música para describir el acuerdo o armonía de los sonidos y lo extrapoló al ámbito de la formación del profesorado. Esto implica que, para que haya congruencia entre lo enseñado en ambas instituciones, todos los tutores (de centros y de universidad) se deban formar en el dominio de las destrezas propias de la profesión. 
Se puede establecer un cierto paralelismo con el modelo de laboratorio o simulación. Exige un nivel de colaboración mayor que el de yuxtaposición, en tanto que los discursos prácticos deben encaminarse a la consecución de unas competencias definidas como importantes por ambas instituciones, además de proporcionar oportunidades de reflexión, estimulación y satisfacción. Sin embargo, uno de los problemas que presenta es que prepara en técnicas o competencias concretas para solucionar cuestiones concretas pero, difícilmente, puede abordar todos los problemas con los que un profesional puede encontrarse. Además, el enfoque investigador del TFM puede verse condicionado negativamente por los profesionales, más centrados en la acción diaria de sus respectivas instituciones.

\section{Modelo de Disonancia Crítica}

Parte de la idea de que las actividades prácticas son difíciles de 'encorsetar' en modelos competenciales. Según este modelo, aprender a ser un profesional requiere ser consciente de lo que se hace y por qué se hace; así como dar razones y reflexionar sobre los orígenes y consecuencias de nuestras conductas y las de los demás. Este conocimiento se ha venido a denominar conocimiento profesional, conocimiento práctico, conocimiento personal (Clandinin y Connelly, citados por Marcelo y Estebaranz, 1998).

Aprender a actuar se entiende como el desarrollo de un aprendizaje experiencial y activo que el estudiante inicia con una serie de teorías y creencias propias, adquiridas antes de comenzar su preparación formal y que se prolonga a lo largo de toda la carrera. Para modificar esas preconcepciones, se intenta que las instituciones promuevan una actitud crítica que provoque un alto grado de disonancia entre lo que se observa en la práctica (a nivel personal, curricular, didáctico, organizativo y social) y sus perspectivas críticas (sobre los aspectos sociales, políticos, históricos y económicos de la profesión).

Este planteamiento es coherente con la esencia del modelo reflexivo, que recoge los planteamientos de Dewey y Schön cuando afirman que el aprendizaje depende de la integración de la experiencia con la reflexión y de la teoría con la práctica. De Dewey destacan los fundamentos del desarrollo potencial de un profesional reflexivo: importancia de la participación activa y deliberada en las situaciones problemáticas, apertura de espíritu, sentido de responsabilidad y dedicación. De Schön, por su parte, enfatizan la relación entre la reflexión y la experiencia y la diferencia entre 'la reflexión en la acción' y 'la reflexión sobre la acción'.

Desde la perspectiva epistemológica, este modelo supone un avance en la reflexión crítica pero no asegura, por sí mismo, la colaboración interinstitucional. De hecho, si es siempre la universidad la que establece unilateralmente el plan de prácticas, puede provocarse un debilitamiento de la cooperación entre las instituciones. 


\section{Modelo de Resonancia Colaborativa}

Más complejo que los anteriores, surge de la necesidad de integrar la formación universitaria de los estudiantes y los programas de aprendizaje con los espacios de aprendizaje: centros de prácticas y universidad. Éstos, como lugares donde se indaga de forma sistemática y crítica, son el elemento más importante dentro de una cultura de colaboración cuyo enlace fundamental es el Practicum y el TFM por las aportaciones de comprensión y mejora que se ofrecen al centro con su desarrollo.

Este modelo se alinea con el modelo de prácticas basado en la investigación sobre la realidad profesional. El futuro formador aprende -guiado por sus tutores y ayudado por sus iguales- indagando y buscando respuesta a sus preguntas (Vaillant y Marcelo, 2001).

Se basa en la idea de que los centros de prácticas deben asumir responsabilidad en la planificación de la formación inicial por medio de la colaboración interinstitucional. Según Marcelo y López (1997), con relaciones de colaboración interinstitucional, no de supremacía, la universidad puede funcionar como una agencia de apoyo a la innovación y al trabajo colaborativo. Para ello se deben seguir los siguientes principios: a) Reciprocidad o intercambio mutuo entre los centros de prácticas y la universidad; b) Experimentación, o disponibilidad de ensayo de nuevas formas de práctica y de estructura organizativa; c) Indagación sistemática; d) Diversidad de situaciones, para que las estrategias se refieran a una amplia variedad de problemas con diferentes características.

\section{La internacionalización, la interculturalidad y la investigación sobre la práctica profesional}

Como se ha visto, tanto el modelo de resonancia colaborativa como el modelo de prácticas basado en la investigación, consideran al profesional en formación como alguien con actitud crítica, reflexiva e innovadora, que orienta su práctica a partir de su propio pensamiento generado desde la búsqueda del conocimiento, de la comprensión y la explicación de lo que sucede en el mundo real.

Así, el conocimiento de la profesión se construye socialmente entre los participantes, de forma que todos aprenden e investigan sobre la práctica. Los estudiantes se convierten en co-aprendices con los profesionales (tutor de la institución, tutor universitario, investigadores) para desarrollar sus conocimientos profesionales y la práctica a medida que van aprendiendo (Goodnough y otros, 2009). Además, el poder es compartido, pues tan importante es el saber, el lenguaje, las críticas o los sustentos teóricos que proporciona el tutor de un centro de prácticas como el de la universidad. Con este planteamiento de aprender juntos, un Practicum reforzado con un TFM puede alcanzar su mayor potencial.

En este trabajo se querría resaltar que estos modelos de prácticas estarían respaldados, además, por las corrientes que preconizan que las necesidades de 
comprensión intercultural, acrecentadas hoy en día por la complejidad proveniente de la globalización, interpelan a todos los profesionales y les obligan a considerar su formación desde componentes de internacionalización, interculturalidad, integrados ambos, en un fuerte corpus de investigación sobre la profesión y sobre la propia práctica (Hudzik, 2011).

La formación de titulados preparados para un mundo global es actualmente una necesidad que debe garantizar la formación universitaria. De hecho, la internacionalización está en las agendas educativas y no sólo de la educación superior, como muestra el hecho de que, ya en la LOE (2006) se hable del propósito de promover la movilidad internacional (refiriéndose a la formación permanente del profesorado).

En general, las fórmulas más trabajadas han sido el fomento de la movilidad de los estudiantes y de los profesionales implicados en actividades formativas, pero la necesidad de formación global lo es para todos los egresados y para todos los profesionales.

Sin embargo, diversos autores avisan de que la simple movilidad no garantiza cambios en los niveles de interculturalidad $y$, por lo tanto, no prepara para comprender la complejidad inherente a la globalización (De Wit, 2011; Vande Berg, Paige \& Hemming Lou, 2012). Una opción complementaria es trabajar la Internacionalization at Home (IaH) y, en concreto, garantizar la 'internacionalización del currículum', para dotar a todos los estudiantes de competencias interculturales e internacionales para ser ciudadanos y profesionales en un mundo global (Beelen, 2007; Leask \& Bridge, 2013).

Si el reto es que los curricula académicos es capacitar a los estudiantes, para que puedan ejercer sus competencias profesionales desde posiciones interculturales, cabe analizar si los estudios incluyen en sus curricula competencias de compromiso ético, responsabilidad social y profesionalidad, así como de conocimientos internacionales relevantes y pertinentes tanto del ámbito profesional como de los contextos en los que éstos se vienen desarrollando. El análisis debe incluir, también, cómo se organiza el currículum para que tales competencias se adquieran de forma eficaz.

\section{El estudio de caso: El Master Erasmus Mundus “Formación de profesionales de la formación"}

\section{El contexto curricular del Practicum y el TFM}

El master Erasmus Mundus "Formación de Profesionales de la Formación" (MUNDUSFOR) se diseña en un momento en que la formación continua se hace indispensable en todos los ámbitos profesionales (educativo, empresarial, administración pública, organizaciones no gubernamentales, etc.) y, sin embargo, ni se ha regulado ni se ha codificado como corpus de conocimiento científico y profesional, suponiendo un vacío en la profesionalización de los formadores. 
Su finalidad es proporcionar una formación universitaria a lo largo de 2 cursos (120 ECTS) a titulados de disciplinas diversas (con un mínimo de 180 ECTS) que quieran orientar su carrera profesional hacia el campo de la formación en empresas, instituciones públicas o privadas e instituciones educativas. Se contempla, por una parte, el perfil del profesional con funciones directas de formación y, por otra, el perfil del profesional que asume responsabilidades de gestión y coordinación de programas, asesoramiento, investigación y evaluación.

Este master se ha impartido durante el periodo 2007-2013 y una vez finalizado, hemos podido comprobar cómo la organización y seguimiento del practicum y del TFM, considerados desde una óptica global, inciden en el proceso de profesionalización según se plantea desde las claves teóricas señaladas. Un elemento imprescindible para que ello ocurra es la coherencia con el resto de módulos desarrollados. Presentamos el diseño general del master para comprender mejor el diseño y desarrollo del Practicum y TFM.

El master MUNDUSFOR, diseñado y desarrollado por un consorcio formado por cinco universidades europeas, ha adoptado la perspectiva internacional, intercultural y profesionalizadora desde su diseño inicial, como se evidencia en la definición de sus diez objetivos formativos:

- Reconocer los aspectos de la formación relativos a los diferentes contextos espacio-temporales y prácticos.

- Reflexionar y tomar posición, de forma crítica, sobre los tipos de formación y sus estrategias en los diferentes países europeos.

- Dominar las técnicas, los instrumentos y los procedimientos de análisis adecuados a los objetivos de la investigación previstos.

- Conocer los modelos de evaluación de la formación y dominar los instrumentos y las técnicas de evaluación.

- Dominar las técnicas y desarrollar una capacidad de reflexión con el fin de organizar, de dirigir y de coordinar acciones formativas de acuerdo con los diferentes contextos europeos de la práctica formativa.

- Analizar los tipos de asesoramiento para adaptarlos a las instituciones y a los contextos formativos.

- Ser capaz de introducir innovaciones tanto en la planificación como en el proceso de formación, teniendo como referencia el comportamiento del grupo.

- Elaborar, desarrollar y evaluar los procesos formativos de acuerdo con los principios de la política formativa de las instituciones educativas, las empresas y las instituciones de los diversos países europeos.

- Concebir su propia práctica profesional como una experiencia generadora de conocimiento y como un objeto de investigación, 
pudiendo participar de esta forma en la profesionalización del formador o de la formadora y mejorar las prácticas profesionales y el ejercicio de la profesión.

- Elaborar una memoria profesional sobre la práctica, integrando las principales etapas de un trabajo de investigación.

La estructura general del master MUNDUSFOR es la siguiente:

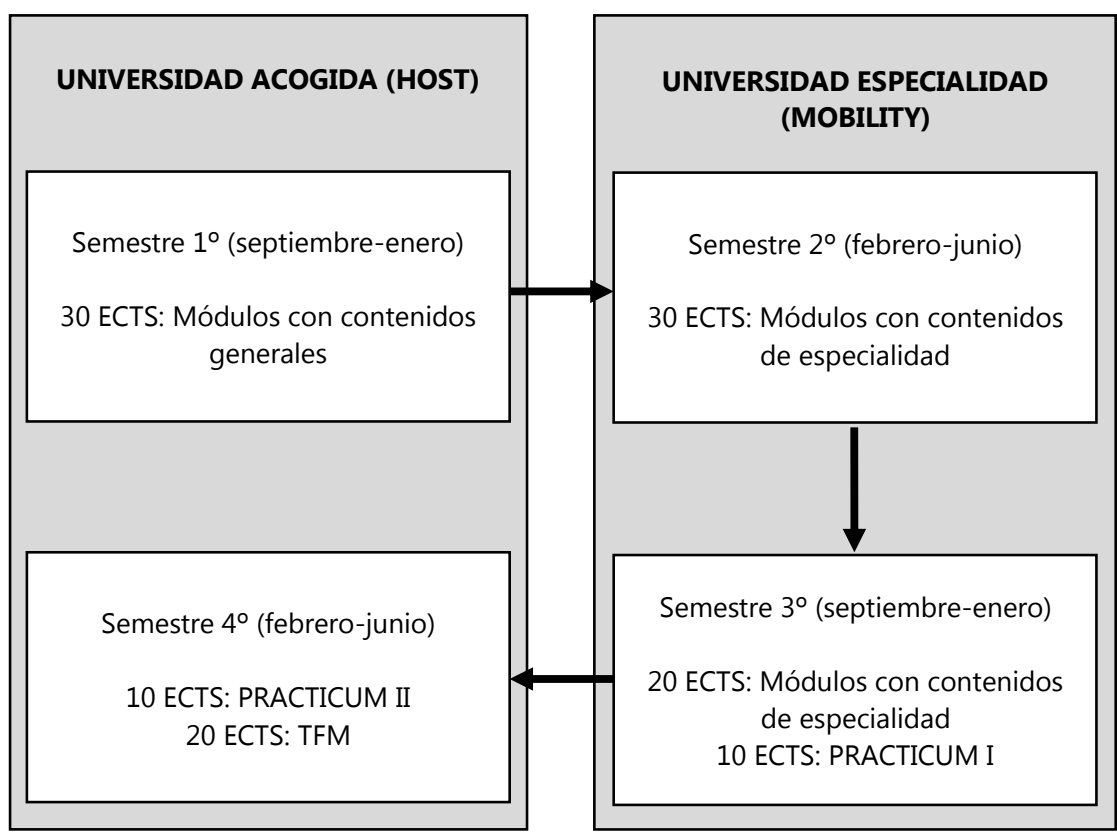

Figura 1. Estructura general del master MUNDUSFOR. Elaboración propia.

Los módulos que se desarrollan atienden a las temáticas siguientes:

- Contextualización y profesionalización

- Evaluación para la mejora de la formación

- Gestión de la formación

- Asesoramiento a instituciones y empresas

- Innovación en formación

- Investigación sobre la práctica profesional de los formadores 
Estas temáticas se abordan, de manera general, durante el primer semestre en lo que llamamos universidad de acogida y se 'profundizan' en las universidades encargadas de desarrollar cada especialidad del master durante el segundo y tercer semestre. Esa progresión en los planteamientos y actividades teórico-prácticas permite al estudiante perfilar las grandes líneas del Practicum y de la investigación que quiere desarrollar en el TFM.

Esto exige una movilidad del estudiante en dos países entre las universidades que forman el consorcio, permitiendo obtener el título oficial conjunto de las cinco universidades europeas ya que hay un tronco común, aunque cada universidad imparte una especialidad:

- Formación de Enseñantes de Formadores en Empresas y de Consultores (Université de Reims Champagne-Ardenne, Francia).

- Formación y Gestión de los Recursos Humanos (Universitat Rovira i Virgili de Tarragona, España).

- Desarrollo Local y Formación de Adultos (Universidade do Porto, Portugal).

- Formación de Formadores para la Enseñanza Técnica y Profesional (Akershus University College, Noruega).

- $\quad$ Formación para la Enseñanza Superior (Universidad de Granada, España).

\section{El diseño del Practicum y del TFM}

El Practicum y el TFM tienen un peso considerable en la formación ofrecida y se conciben como materias totalmente integradas en el conjunto del master. Constituyen el eje sobre el que pivota la titulación, ya que es donde se movilizan las competencias propias de esta formación y por ello constituyen, además, momentos propicios para evaluarlas, especialmente por tratarse de un master eminentemente profesionalizador. Las competencias del Practicum y del TFM son:

- Conocer el papel de la formación en Europa y en el mundo.

- Desarrollar una postura de búsqueda sobre la práctica de la formación (reflexión 'en' la práctica y 'sobre' la práctica) en diferentes ámbitos con el fin de mejorar el ejercicio profesional del formador.

- Desarrollar una postura de trabajo en equipo en las situaciones propias de los contextos profesionales de las prácticas.

- Dirigir y gestionar los servicios de formación de empresas, de administraciones públicas o de instituciones educativas.

- Diseñar, desarrollar y evaluar procesos de intervención formativa.

- Elaborar informes reflexivos sobre los propios procesos desarrollados durante las fases del Practicum. 
Entendemos que el Practicum y el TFM son espacios privilegiados que ofrecen oportunidades y experiencias de inserción a la realidad profesional, de profesionalización y de mejora del ejercicio de la formación. Se constituyen, a la vez, como:

- Contenido formativo, en tanto que aportan conocimientos prácticos sobre el ejercicio profesional.

- Estrategia formativa, ya que facilitan contrastar la teoría y la práctica profesional, así como generar conocimiento propio mediante la reflexión e investigación sobre la práctica al enfrentarse a problemas reales de la profesión.

- Proceso evaluativo: Desde la doble perspectiva formativa y sumativa/acreditativa (autoevaluación del propio estudiante y heteroevaluación).

La convivencia y experiencia en ámbitos profesionales, desde el desarrollo de funciones asociadas a la formación, permite captar la complejidad del mismo desarrollo profesional y la necesidad de formación permanente. Se abordan para ello estrategias de observación participativa y reflexión 'en' la práctica y 'sobre' la práctica; coordinación intra-equipos y trabajo en red con otros profesionales; evaluación de procesos; y autoevaluación y transferencia de los propios aprendizajes. La intención es favorecer la inserción en relación con su perfil profesional, permitir su desarrollo personal, así como la innovación social.

El Practicum (20 créditos) comporta un mínimo de 500 horas de trabajo para el estudiante, repartidas de la manera siguiente: Un mínimo de 300 horas de Practicum en los centros escogidos (150 horas en cada país) y el resto (200 horas) se dedican a las tutorías, individuales y grupales, así como a la elaboración de la memoria de prácticas (una para cada país).

El TFM (20 créditos) se desarrolla formalmente durante el cuarto semestre del master (figura 1). Sin embargo, desde el inicio de prácticas, los estudiantes y tutores toman decisiones sobre la temática, foco, propósitos, etc. De hecho, los tutores de la universidad de la especialidad acompañan el proceso de realización del TFM desde el primer periodo de prácticas hasta su defensa (figura 2). 


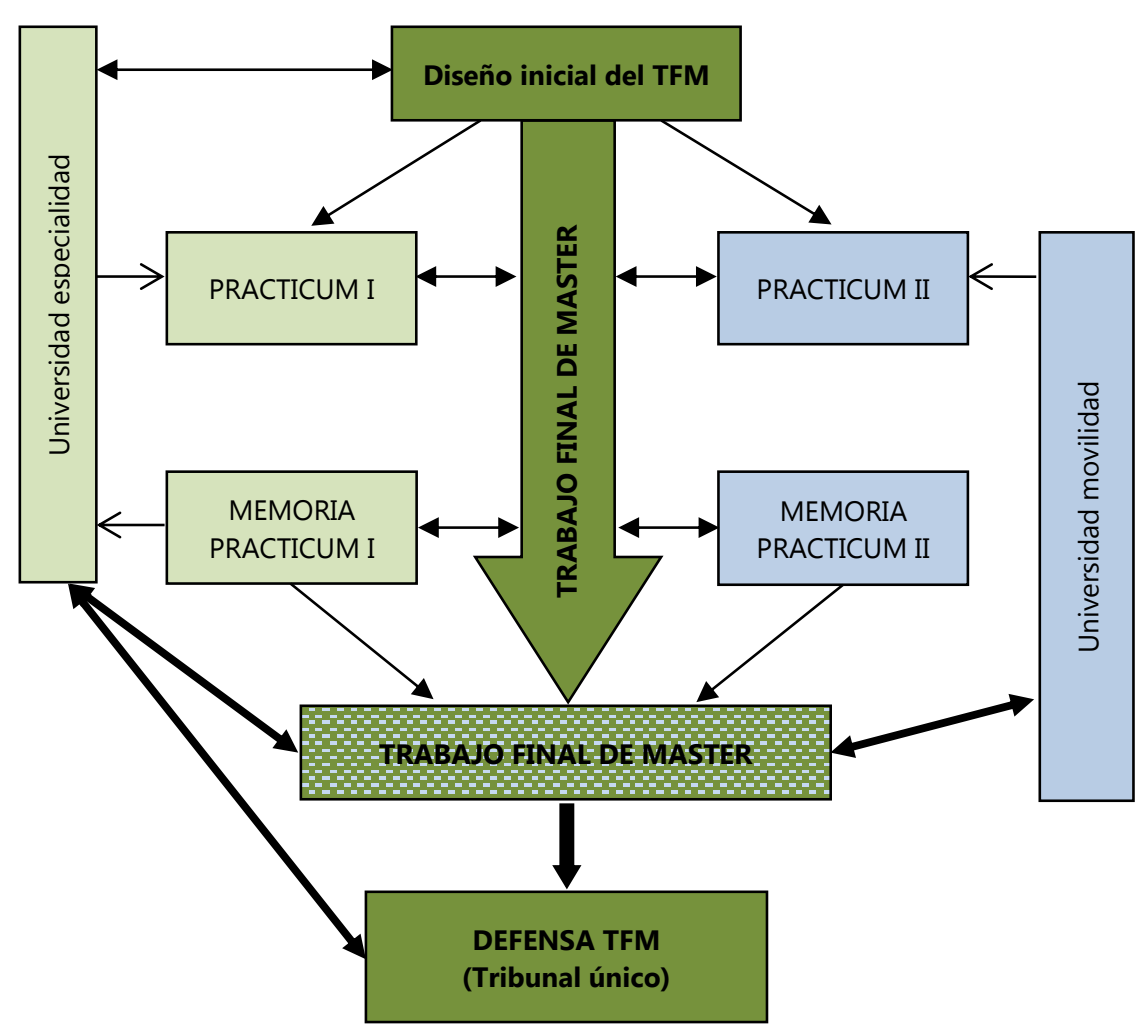

Figura 2. Relación entre el Practicum (I y II) y el TFM en el master MUNDUSFOR. Elaboración propia.

El requisito de realizar el Practicum dos instituciones de diferente país, aun con la complejidad que le confiere, le dota de una gran riqueza porque obliga a contrastar contextos profesionales, desde una perspectiva internacional e intercultural, objetivo fundamental e indisociable de este master.

No podemos olvidar que la profesión del formador se desarrolla en contextos con culturas sociales, económicas, profesionales, laborales, educativas... diferentes, dependiendo del ámbito donde se está formando. Veremos los ámbitos y temáticas emergentes que se han abordado (figura 4).

Por otra parte, la movilidad laboral actual exige una formación que potencie la capacidad de ejercer cualquier profesión atendiendo y comprendiendo las necesidades del propio contexto, pero con una visión amplia. Ésta la ofrece el conocimiento de esa misma profesión en contextos sociogeográficos diferentes, favoreciendo competencias para, lo que ha venido a llamarse, un mundo "glocal", donde cada vez es más necesario pensar globalmente y actuar localmente. 


\section{Momentos clave en la profesionalización}

Los estudiantes que acceden al master provienen de una formación inicial muy diversa y tienen un bagaje académico y profesional, en ocasiones, alejado de los ámbitos formativos. Por eso, se hace imprescindible que desde el inicio del master, con el desarrollo de los módulos, se creen las condiciones para que el estudiante comprenda la profesión de formador y vaya construyendo una identidad profesional específica: primer momento de profesionalización. En este momento debe alcanzar la conciencia de que el ejercicio de la profesión comporta, además de unos conocimientos específicos, una reflexión crítica sobre la interacción que se establece entre el contexto concreto y su propia actuación, basada en el análisis de evidencias.

Así es cómo, en el tercer semestre, el estudiante se introduce en un contexto laboral en el que deberá poner en marcha los conocimientos y estrategias que, bajo la orientación y guía del tutor de Practicum y de TFM, le permitan desarrollar una actuación profesional a la vez que se le demanda una recogida de evidencias y análisis de las mismas. Por ello se inicia un segundo momento de profesionalización, en el que debe:

- Centrar el eje de su especialización profesional, a partir de unas prácticas y una investigación en un ámbito laboral determinado.

- Definir las bases teóricas para fundamentar las prácticas y la investigación.

- Concretar las acciones a realizar en el centro para conocer el contexto y para intervenir e investigar en el mismo.

- Plantear las bases metodológicas para dar cumplimiento al Plan de Prácticas y diseño del TFM.

En el cuarto semestre deberá contrastar dos realidades profesionales en países diferentes. Los dos periodos de prácticas se desarrollan en el mismo ámbito profesional, para que puedan abordar e investigar la misma temática. Por ejemplo, si el objeto de investigación es la formación de los formadores que trabajan con colectivos inmigrantes, las prácticas podrían hacerse en un centro de educación formal (Practicum I) y en una organización no gubernamental (Practicum II).

De ahí que afirmemos que se inicia un tercer momento de profesionalización, ya que exige al estudiante replantear su profesión de formador a partir del conocimiento de una nueva realidad, debiendo poner en cuestión patrones establecidos de actuación a partir de un proceso de investigación sobre su propia práctica.

La elaboración de un informe de investigación (TFM) y defensa pública comporta el cuarto momento de profesionalización, ya que:

- Posibilita un proceso completo de investigación sobre la práctica profesional: problematización, movilización de referencias teóricas, establecimiento de rigor metodológico, análisis de los datos, propuesta de solución de problemas y elaboración de conclusiones. 
- Desarrolla las competencias comunicativas al hacer una defensa pública del trabajo en un idioma distinto al que se ha escrito.

- Ayuda a apreciar y a evaluar las dificultades, las debilidades y las fortalezas en sus tareas de investigación, a partir de la tutorización seguida y de las consideraciones del tribunal.

Es necesario insistir en el papel de guía/orientador del tutor universitario en el proceso de profesionalización, acompañado por el tutor de las instituciones colaboradoras. De ahí la importancia de la selección de instituciones y ámbitos que participan.

\section{El desarrollo del Practicum y del TFM: Definición de ámbito y temática}

El Practicum y TFM se desarrolla a partir de una organización que integra los objetivos del master, los intereses de los estudiantes y las necesidades de las instituciones de prácticas (figura 3).

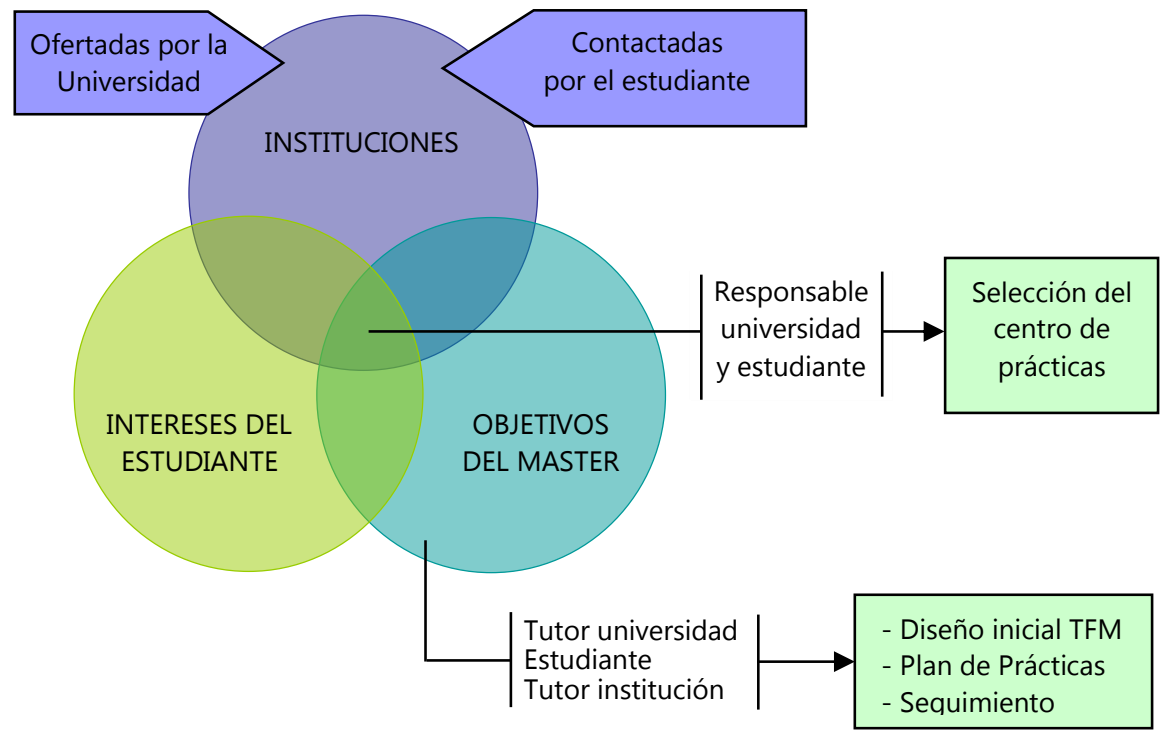

Figura 3. Definición del ámbito y la temática del Practicum y el TFM en el master MUNDUSFOR. Elaboración propia.

A partir de esta organización cada universidad tiene unos requerimientos específicos a la hora de establecer la relación con los centros de prácticas. Por ejemplo, hay sedes que exigen un convenio previo entre la universidad y el centro de prácticas, 
mientras que, en otras, el Practicum se puede desarrollar con un contacto de tipo menos formal. Algunas universidades tienen una oferta de centros colaboradores para que los estudiantes elijan el lugar donde realizar las prácticas; en otras, son los estudiantes los que buscan y hacen los contactos previos con las empresas o instituciones. Otras conjugan ambas posibilidades.

En cualquier caso, es fundamental el papel del responsable de cada sede para determinar las posibilidades formativas de dichas instituciones y facilitar la incorporación de los estudiantes. Así mismo, es esencial la labor de los tutores de la universidad y de la institución que acompañan el proceso del plan de prácticas. Esencial es el papel del tutor en la universidad de la especialidad, ya que además de esas funciones dirige también el TFM, en el que deben confluir los dos periodos de prácticas.

Por otra parte, los estudiantes deben adoptar una postura de profesional en formación, ejerciendo así un doble rol: por un lado, involucrándose en la actividad cotidiana del centro de prácticas y, por otro, investigando un aspecto concreto de la función formativa en el que confluyen sus intereses y las necesidades manifestadas por la institución.

A partir de este proceso tan abierto, a lo largo de las diferentes ediciones del master, han aparecido una gran diversidad de espacios de trabajo y análisis que hemos clasificado en cuatro grandes ámbitos del Practicum y el TFM (universidad, administración pública y empresa, entidades sociales y culturales, y centros educativos) y en cada uno de ellos se han reflejado las temáticas, agrupadas por subámbitos (figura 4). Se trata -entendemos- de respuestas que el master da a necesidades emergentes de la sociedad y que ayudan a vislumbrar el campo de la formación como un ámbito aún por desarrollar en muchos sentidos. 


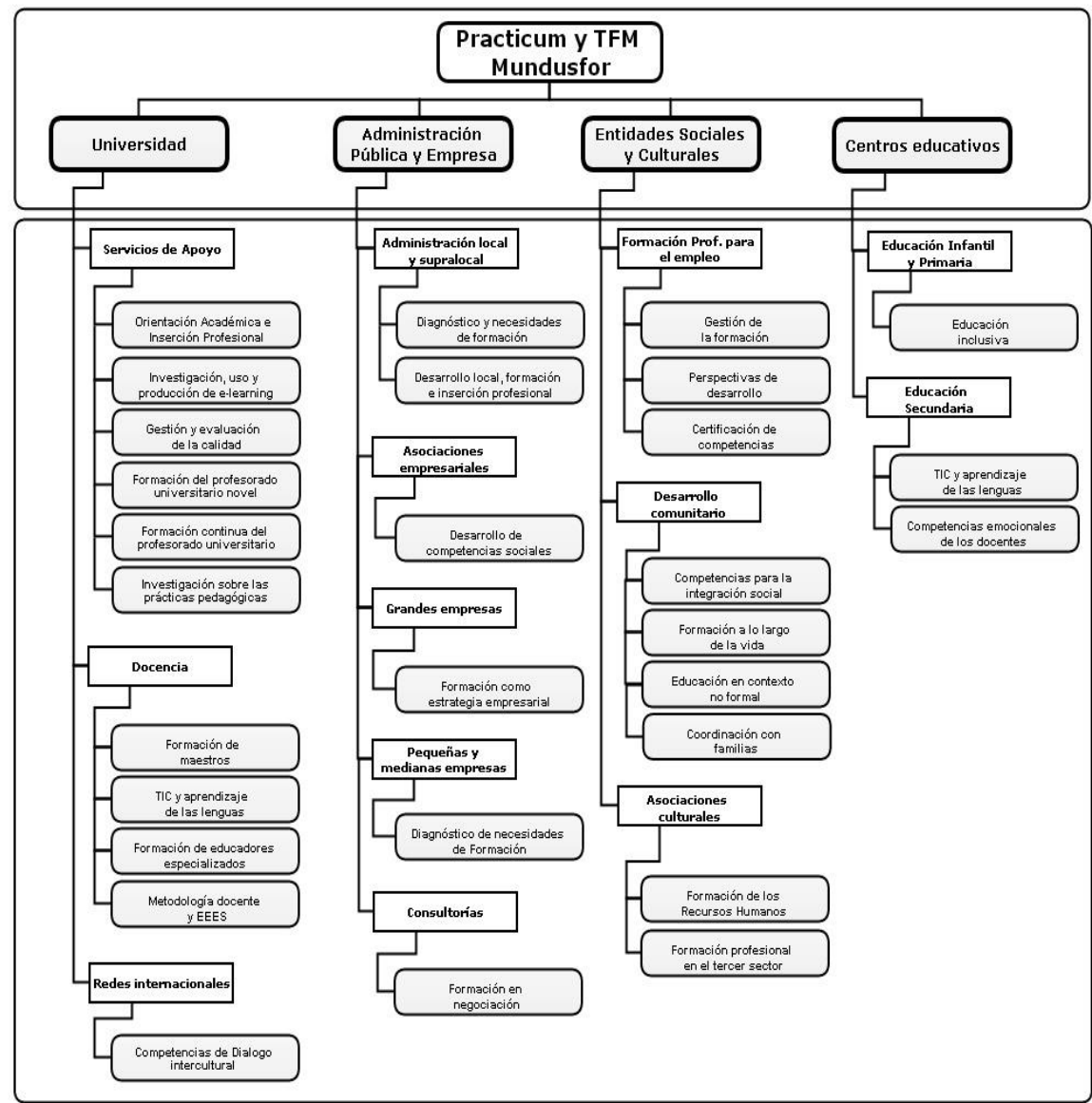

Figura 4. Ámbitos y temáticas abordados en el Practicum y el TFM por las diferentes cohortes del master MUNDUSFOR. Elaboración propia.

\section{El proceso de evaluación}

Cuando se habla de evaluación en procesos didácticos, se suele plantear la evaluación de los aprendizajes de los estudiantes. En este trabajo, este aspecto lo abordamos someramente para detenernos en la evaluación que los estudiantes hicieron del master en su globalidad, con la intención de analizar la consecución de sus objetivos y en coherencia con el planteamiento de la necesidad de un análisis y reflexión crítica como base de la profesionalización del formador.

En lo referente a la evaluación de los aprendizajes de los estudiantes, el Practicum y TFM conceden mucha importancia a la evaluación formativa y continua, como se 
observa a través de lo ya expuesto pero, obviamente, también reconocen la función acreditativa de la evaluación que se plantea de la siguiente manera:

En el Practicum se tiene en cuenta la implicación de los estudiantes en el seguimiento del trabajo (10\%), así como el proceso y la memoria de prácticas, tanto por parte del tutor universitario (60\%) como por el del centro de prácticas (30\%).

En el TFM se consideran aspectos formales referidos a la redacción y adecuación a las normas de presentación (10\%), aspectos de contenido como la fundamentación teórica (20\%), la definición y adecuación metodológica (20\%), el análisis de resultados (20\%) y la exposición y defensa pública del trabajo (30\%).

En lo referente a la evaluación del master se han seguido los protocolos establecidos por las diferentes normativas de cada país y las directrices que corresponden a la EACEA (Agencia Ejecutiva en el ámbito Educativo, Audiovisual y Cultural) de la Comisión Europea. Además, cada sede ha arbitrado sistemas de seguimiento propios para la evaluación del master por parte de los estudiantes.

En la Universitat Rovira i Virgili se ha optado por un cuestionario abierto en el que los estudiantes analizan algunos aspectos sobre el diseño y desarrollo del Practicum y TFM (organización y coordinación; tutoría y seguimiento por parte de la universidad y la institución; aportaciones a su formación profesional). También realizan un autoinforme en el que valoran la aportación del Practicum y TFM a su formación personal y profesional. De los resultados que ofrece el análisis de las respuestas ofrecidas por los estudiantes de diferentes promociones, destacamos los siguientes:

Desde el punto de vista curricular, aparece como una condición de calidad la clarificación integral del proceso de Practicum y de la tutorización desde su inicio. La integración del Practicum en el plan de estudios se considera adecuada y se resalta, sobre todo, la idoneidad de las tutorías académicas tanto individuales como grupales:

Estoy muy satisfecha, también, con la tutoría recibida en la universidad. Fue una relación equilibrada entre la libertad para moverme y trabajar de acuerdo a mis intereses y el apoyo y asesoramiento para allanar el camino que emprendía. (AIR)

La visión global del proceso estuvo a cargo de la coordinación que realizó una excelente labor de seguimiento con reuniones mensuales grupales y asesoramiento personal permanente...el soporte que los alumnos tuvimos inclusive de los tutores que no eran los propios... (MB)

Las tutorías recibidas en las instituciones son bien valoradas, en general, aunque, en algunos casos, reclaman mejorar la atención prestada tanto para el conocimiento de la institución como para la realización de la memoria de Practicum. Algunos estudiantes experimentan la diversidad de concepciones e intereses profesionales entre los ámbitos académico y profesional y lo valoran también como formativo. 
A nivel organizativo, se valora muy positivamente el haber previsto las instituciones tras consultar las preferencias de los estudiantes, por lo que la adecuación a los intereses profesionales es mayor:

La coordinación de Mundusfor en Tarragona pidió con amplia anticipación información referida a las primeras prácticas realizadas, el tema de investigación e intereses para la segunda práctica...mis prácticas fueron concertadas mucho antes de mi ingreso en Tarragona...(ZR)

En cuanto a las aportaciones a nivel profesional, los aprendizajes que resaltan son el haber dado solidez a sus ámbitos profesionales o de formación iniciales por medio del contacto con la realidad laboral, particularmente en el ámbito de los recursos humanos. Hablan explícitamente de la complejidad percibida en el desempeño profesional. También se subrayan aspectos como el compromiso, responsabilidad, climas de trabajo, etc. vividos en las instituciones y en contacto con personas relevantes en el entorno profesional. Esto les ha ayudado a observar en la práctica los aspectos morales de la profesión. En algún caso, incluso se habla de regeneración de esperanza social en contraste con las experiencias en su país de origen:

...he percibido y recuperado en mí que los sueños estaban escondidos pero no muertos y que, otra vez, florecieron como una posibilidad real de creer en algo mejor para la humanidad. Después de años trabajando en esto...y bastante desanimada por las dificultades encontradas pude ver que todavía es posible... (MM)

Resalta la importancia que otorgan al Practicum en la construcción del conocimiento y el tratamiento científico de los datos e informaciones de que disponen las instituciones. Muestran la necesidad de mejorar su conocimiento sobre el tratamiento de la información y sobre la elaboración de informes científicos sobre su práctica.

También sería remarcable la importancia concedida a aspectos como la interculturalidad e internacionalización, el uso de las TIC, las estrategias de comunicación y el dominio de idiomas, como elementos imprescindibles de la profesionalización.

Por último, muestran deseos de ampliar su experiencia y profundizar en la profesionalización para abordar proyectos de desarrollo local, la relación entre educación formal y no formal, la dirección de recursos humanos, la relación entre formación y TIC e, incluso en continuar su formación con un doctorado.

Desde el punto de vista de su desarrollo personal, destacan un mayor autoconocimiento de sus fortalezas y debilidades. Las fortalezas serían su implicación, capacidad de trabajo y motivación para el aprendizaje, así como una mayor conciencia de la importancia de las relaciones intra e interinstitucionales y los ámbitos del desarrollo profesional: 
...esta experiencia me ha dejado más consciente y atenta a los desafíos de ocupar un cargo con tanta responsabilidad (el de formador de formadores) y exige sobre todo compromiso social y ético. (LL)

Las debilidades aluden a aspectos como la gestión del tiempo, la resolución de conflictos, la gestión del proceso de investigación incluyendo la realización de informes:

...intentaré hacerme competente en la organización de la investigación, puesto que para lo práctico ya lo soy. (MdL)

...se me dificulta trabajar individualmente en informes largos. (ZR)

...reflexiono sobre mi actitud frente a esa situación y pienso que tal vez podría haber actuado diferente, (...) si hubiese reaccionado más deprisa podría haber hecho el cuestionario, pero dejé que siguiera su curso y sus tiempos de acuerdo a la dinámica institucional... previendo esta cuestión, sabiendo que lo que allí estaba actuando es parte de la 'dimensión política' de la formación. (AIR)

\section{Conclusión}

Desde el punto de vista del carácter marcadamente profesionalizador otorgado al Practicum y al TFM en este master, cabe destacar las siguientes ideas-clave:

La integración de competencias específicas y, sobre todo, generales ha de potenciar el desarrollo polivalente de los estudiantes a partir de la profundización en sus propios itinerarios profesionales. También debe haber una fuerte articulación curricular de los dos periodos de prácticas con el TFM, así como con el resto de módulos. En ese sentido defendemos un diseño holístico de formación profesionalizadora (ilustrado en las figuras 1 y 2 , respectivamente) centrado en procesos de 'inserción profesional' a partir de situaciones reales. En ellas concurren competencias propias de la intervención profesional que constituyen el eje del Practicum y de la investigación vinculada a la práctica canalizada a través del TFM.

En ese proceso, el papel de las tutorías individuales y grupales (tanto en la universidad como en las empresas o instituciones) es indiscutible aunque debemos señalar la necesidad de informar y formar a los tutores para que asuman un rol activo y optimizar, así, su implicación. El responsable de la organización del Practicum y TFM debe velar por la adecuación de las instituciones y la formación de los tutores para la consecución de los objetivos del master, ya que, como en todos los procesos de inserción profesional, la acogida emocional es prioritaria (Negrillo e Iranzo, 2009). Al mismo tiempo, han de incentivar la amplitud de perspectivas, a partir de la libertad para escoger ámbitos y temáticas, de manera que la profesión del formador vaya ampliando y profundizando su campo de estudio y actuación. 
También se precisan procesos de evaluación y regulación que evidencien la consecución de las competencias acordes con el ámbito profesional. Como se sabe, la investigación reciente pone de manifiesto que la universidad forma exitosamente en competencias de naturaleza 'conceptual-declarativa'; puede hacerlo también, si bien en menor grado, en el caso de las 'técnico-procedimentales' $y$, aunque las contempla, aparecen serias dificultades ante la formación de las competencias verdaderamente 'estratégicas' como interpretar, decidir, emprender acciones, etc., ampliamente afectadas por el conocimiento contextual y profesional. Por su parte, los ámbitos profesionales no formarían en competencias de investigación, habilidades de supervivencia sociopersonal o procesos de toma de decisiones complejos, ni en perspectivas comparadas de carácter internacional (Monereo y Pozo, 2005).

Por ello, además de precisarse la construcción compartida de las competencias entre los ámbitos académico y profesional, la formación universitaria debe trabajar en la creación de contextos experienciales más cercanos al 'saber hacer' profesional permitiendo que los estudiantes ejerciten competencias integrales y complejas como las planteadas. Acompañar la construcción de conocimiento científico y profesional requiere problematizar la práctica en la que los estudiantes son protagonistas.

En ese sentido, en el estudio de caso que presentamos se pone de manifiesto que el tándem que forma el Practicum y TFG es un escenario privilegiado para trabajar y evaluar todo tipo de competencias, incluso éstas últimas. Esto se refuerza con la realización de los dos Practicum en diferentes países, momentos y contextos culturales, que enriquece el proceso al permitir al estudiante analizar su propia realidad (la de su país de origen) desde perspectivas diferentes, incorporando a su bagaje personal y profesional elementos de transferencia del conocimiento, internacionalización e interculturalidad del todo imprescindibles (Ferrer, Carmona y Soria, 2012). Ahora bien, deben considerarse determinadas condiciones curriculares, organizativas y personales que aseguren su sentido formativo. Por ejemplo, se percibe como una fortaleza el hecho de decidir los lugares de prácticas con suficiente antelación para facilitar al estudiante la contextualización previa y reducir su tiempo de adaptación, así como atender sus intereses para potenciar su implicación y responsabilidad.

Finalmente, se constata la necesidad de robustecer el corpus teórico-práctico en torno a la figura del formador. Las temáticas abordadas en el Practicum y el TFM (figura 4) son el resultado de conjugar los intereses de los estudiantes con los de las instituciones de prácticas. En ese sentido, pueden considerarse respuestas a necesidades profesionales y sociales de gran calado y actualidad. Independientemente del ámbito y la temática, el profesional de la formación está asumiendo, aun con lagunas formativas importantes, funciones emergentes que giran en torno a nuevas formas de gestión y gobernanza, evaluación institucional, investigación, desarrollo local, certificación de competencias, etc. Todo ello pone de manifiesto que el formador constituye una figura profesional necesaria, en alza y en evolución y que, por lo tanto, necesita de formación específica que priorice procesos 
de investigación y de evaluación comprometidos con la ética profesional y la predisposición a la mejora continua.

\section{Notas}

${ }^{1}$ Este artículo se enmarca en el contexto del Master Erasmus Mundus "Formación de profesionales de la formación" (MUNDUSFOR), aprobado en la convocatoria 2006 por la EACEA (Agencia Ejecutiva en el ámbito Educativo, Audiovisual y Cultural) de la Comisión Europea. Inscrito en el Registro de Universidades, Centros y Títulos (RUCT) con el código 3001542.

\section{Referencias bibliográficas}

Agència per a la Qualitat de Sistema Universitari de Catalunya [AQU] (2013). Com elaborar, tutoritzar i avaluar un Treball de Fi de Màster, Barcelona: AQU.

Beelen, J. (Ed.) (2007). Implementing Internationalisation at Home, EAIE Professional Development Series for International Educators, Amsterdam: European Association for International Education.

Cochran-Smith, M. (1999). Reinventar las prácticas de Magisterio. En A. Pérez, J. Barquín y J.F. Angulo (Eds.), Desarrollo Profesional del Docente. Política, investigación y práctica (pp. 533-552). Madrid: Akal.

De Wit, H. (2011). Internationalization of Higher Education: Nine Misconceptions. International Higher Education, 64, 6-7. Recuperado de: https://htmldbprod.bc.edu/pls/htmldb/f?p=2290:4:0::NO:RP,4:P0_CONTENT_ID:11511 $\underline{7}$

Denzin, N.K. (2007). On Understanding Emotion, New Brunswick (N.J.): Transaction Publishers.

Domingo, R. y Fuertes, M.T. (2007). Hacia un modelo holístico del practicum en el marco del EEES. Ponencia presentada en el IX Symposium Internacional del Practicum, Poio, 27-29 junio.

Ferrer, V.; Carmona, M. y Soria, V. (Eds.) (2012). El trabajo de fin de grado. Guía para estudiantes, docentes y agentes colaboradores, Madrid: McGraw-Hill.

Gardner, H. (1998). Inteligencias múltiples: la teoría en la práctica, Barcelona: Paidós.

Goleman, D. (1996). Inteligencia emocional, Barcelona: Kairós.

- (2005). La práctica de la inteligencia emocional, Barcelona: Kairós.

Goodnough, K. y otros (2009). Exploring a triad model of student teaching: Preservice teacher and cooperating teacher perceptions. Teaching and teacher education, 25 (2), 285-296. 
Hudzik, J. K. (2011). Comprehensive Internationalisation. From Concept to Action, Washington: NAFSA.

Jorro, A. (2007). L'alternance recherche-formation terrain professionnel. Recherche et formation, 54, 101-114.

Leask, B. \& Bridge, C. (2013). Comparing internationalization of the curriculum in action across disciplines: theoretical and practical perspectives. Compare: $A$ Journal of Comparative and International Education, 43 (1), 79-101. Recuperado de: http://www.tandfonline.com/doi/abs/10.1080/03057925.2013.746566

Marcelo, C. y López, J. (1997). Asesoramiento curricular y organizativo en Educación, Barcelona: Ariel.

Méndez, L. (2012). El conocimiento situado y los sistemas de actividad. Un modelo teórico para re-pensar el Practicum. Revista de Educación, 359, 629-642.

Ministerio de Educación y Ciencia (2006). Ley Orgánica 2/2006, de Educación (LOE), de 3 de mayo de 2006. BOE (4.5.2006).

- (2007). Real Decreto 1393/2007, por el que se establece la ordenación de las enseñanzas universitarias oficiales, de 29 de octubre de 2007. BOE (30.10.2007).

Monereo, C. y Pozo, J. I. (Coords.) (2005). La práctica del asesoramiento a examen, Barcelona: Graó.

Negrillo, C. e Iranzo, P. (2009). Formación para la inserción profesional del profesorado novel de Educación Infantil, Educación Primaria y Educación Secundaria: hacia la reflexión desde la inducción y el soporte emocional. Profesorado. Curriculum y formación del Profesorado, 13 (1), 1-26. Recuperado de: http://www.ugr.es/ recfpro/rev131ART11.pdf.

Orland-Barak, L. (2006). Tutores o mentores que aprenden a participar en discursos competitivos de la práctica educativa. Revista de Educación, 340, 187-212.

Schön, D. (1998). El profesional reflexivo: cómo piensan los profesionales cuando actúan, Barcelona: Paidós/MEC.

Tejada, J. (2005). El trabajo por competencias en el Practicum: cómo organizarlo y cómo evaluarlo. Revista Electrónica de Investigación Educativa, 7 (2). Recuperado de: http://redie.uabc.mx/vo7no2/contenido-tejada.html

Vaillant, D. y Marcelo, C. (2001). Las tareas del formador, Archidona: Aljibe.

Vande Berg, M.; Paige, R. M. \& Hemming Lou, K. (Eds.) (2012). Student learning abroad: What our students are learning, what they're not, and what we can do about it, Sterling (Va.): Stylus.

Zabalza, M.A. (2004). Condiciones para el desarrollo del Practicum. Profesorado. Revista de Currículum y Formación de Profesorado, 8 (2), 1-22. Recuperado de: www.ugr.es/local/recfpro/Rev82.html 
- (2007). El Practicum y las prácticas en empresas en las coordenadas de la convergencia. En M. Raposo y otros (Coords.), El Practicum en el nuevo contexto del Espacio Europeo de Educación Superior (pp. 63-87). Santiago de Compostela: Universidad de Santiago de Compostela.

- (2011). El Practicum en la formación universitaria: estado de la cuestión. Revista de Educación, 354, 21-43.

Zabalza, M.A. y Marcelo, C. (Coords.) (1993). Evaluación de Prácticas: Análisis de los procesos de formación práctica de los profesores de primaria y media de la Universidad de Sevilla, Sevilla: GID.

\section{Nota biográfica}

Charo Barrios Arós es profesora titular de universidad del Departamento de Pedagogía de la Universitat Rovira i Virgili y ha sido Responsable del Máster Formación de Profesionales de la Formación (URV). Investiga en docencia universitaria, formación inicial y permanente y mejora escolar.

Pilar Iranzo García es profesora y Responsable del Grado de Educación Infantil en la Universidad Rovira i Virgili (Tarragona). Investiga en docencia universitaria, formación inicial y permanente, aprendizaje adulto y mejora escolar. Es autora de Innovando en educación. Formarse para cambiar: un viaje personal (2009) y de Asesoramiento pedagógico al profesorado (2012).

Juana María Tierno García es profesora agregada del Departamento de Pedagogía y Responsable del Grado de Educación Social de la Universitat Rovira i Virgili. Sus principales líneas de investigación son la evaluación y la investigación sobre la docencia universitaria y el liderazgo y la mejora escolar.

Datos de contacto:

Universidad Rovira i Virgili. Departamento de Pedagogía.

Facultad de Ciencias de la Educación i Psicología. Ctra. de Valls, s/n 43007-Tarragona

E-mail: charo.barrios@urv.cat, pilar.iranzo@urv.cat, juanamaria.tierno@urv.cat 